\title{
Ecological Production of Cores - Example from Automotive Branch
}

\author{
Marko Grzinčič ${ }^{1}$, Petr Hlavsa ${ }^{2}$ \\ ${ }^{1}$ Private address: Mozartova 23, 46001 Liberec 1, The Czech Republic, E-mail: mgrzincic@yahoo.de \\ ${ }^{2}$ Process Engineering, Nemak Slovakia Ltd., Ladomerská Vieska 394, 96501 Žiar nad Hronom, The Slovak Republic, E- \\ mail: petr.hlavsa@nemak.com
}

The paper informs readers about the latest trends in ecological production of cores for the most demanding castings made of aluminum alloys on example of cylinder heads for combustion engines of the passenger cars. Although the cores bonded with silicates are known since the 60th years of the 20th century, only in recent years the technical progress has undergone such level that the leading producers of cylinder heads are able to deploy the system in serial production. Nemak started solving inorganic systems 12 years ago and today it delivers selected products to its customers and during their production there are cores being used which are joined by ecologic systems. The contribution analyses limiting technical aspects at application of inorganic systems. They replace systems of cold-box and hot/warm-box and it presents in details the benefits of ecological solutions which may be found in final product features. At the same time, it also points at difficulties which are still necessary to be solved.

Key words: casting, core, binder, inorganic, ecology

\section{References}

[1] IDEN, F., TILCH, W., WOJTAS, H.J. (2011). Der Sand als Einflussfaktor auf die kern- und gießtechnischen Eigenschaften von Cold-Box-Kernen. Giesserei, 98. year, No. 9, pp. 18-25. Germany.

[2] JELÍNEK, P. (2004). Pojivové soustavy slévárenských formovacích směsí (Chemie slévárenských pojiv), ISBN 80-239-2188-6. Ostrava

[3] PABEL, T., KNEISSL, CH., BROTZKI, J., MÜLLER, J. (2011). Verbesserte mechanische Eigenschaften von AlGussteilen durch den Einsatz von INOTEC ${ }^{\circledR}$ Kernen. Giesserei-Rundschau, 58. year, No. 1-2, pp. 20-29. Austria.

[4] WEISSENBEK, E., WILLIMAYER, J., WOLF, J. (2008). BMW-Leichtmetallgießerei setzt auf anorganisch gebundene Kerne. Giesserei, 95. year, No. 6, pp. 32-35. Germany.

[5] BOEHM, R., LÖCHTE, K. (2013). Cordis Bindersystem, International Conference Foundry Moulding Materials, Kurdějov, Czech Republic.

[6] LÖCHTE, K., BÖHM, R. (2005). Cordis. The inorganic binder system - properties and experience. Casting plant + Technology International, No. 3, pp. 6-11. Germany.

[7] MÜLLER, J. (2012). Inorganic Binder System Sandwiched Between „Technology Push” and “Market Pull”. Giesserei, No. 2, pp. 52-58. Germany.

[8] MÜLLER, J. (2013). Geht nicht, gibt's nicht - Neue Entwicklungen im Bereich anorganischer Bindersysteme. German Foundry Day, Fellbach.

[9] MÜlLER, J. (2013). Geht nicht, gibt's nicht. Giesserei, No. 8, pp. 104-105. Germany.

[10] http://www.sandteam.cz/nase-sluzby/geopolymery

[11] STEINHÄUSEr, T., WEHREN, B., WIESAUER, J. (2008). Einsatz anorganischer Binder in der neuen Kokillengießerei der MWS Casting s.r.o./Slowakei. Giesserei, 95. year, No. 6, pp. 66-68. Germany.

[12] http://www.awb-online.com/en/start.htm

[13] LÖCHTE, K. (2013). Cold-Box - technologie s budoucností. Slévárenství, No. 9/10. Czech Republic.

[14] WALLENHORST, C. (2010). Chemische Grundlagen der anorganischen Bindemittel - Betrachtung der Kernherstellung auf molekularer Ebene. Giesserei-Rundschau, 57. year, No. 3-4, pp. 50-52. Austria.

[15] FALLER, M. (2010). Anorganische Kernherstellung - Umsetzung in der Anlagentechnik. Giesserei-Rundschau, 57. year, No. 1-2, pp. 8-10. Austria.

[16] BÖHM, R., ASAL, J., MÜNKEL, B. (2013). Der Weg zu einer wirtschaftlichen und emmisionsfreien Gießerei. Giesserei, 100. year, No. 1, pp. 68-73. Germany.

[17] LÖCHTE, K., BÖHM, R. (2005). Cordis. Das anorganische Bindemittelsystem - Eigenschaften und Erfahrungen. Giesserei, 92. year, No. 3, pp. 68-71. Germany. 
[18] MÜLLER, J. (2012). Economic and environmental benefits of Inotec in serial production. Casting Plant \& Technology, No. 1, pp. 6-8. Germany.

[19] KAUTZ, T., WEISSENBEK, E., BLÜMLHUBER, W. (2010). Anorganische Sandkernfertigung: ein Verfahren mit Geschichte. Giesserei, 97. year, No. 08, pp. 66-69. Germany.

[20] SCHWICKAL, H., HOFFMANN, H., BLÜMLHUBER, W., WEISSENBEK, E. (2009). Regenerierung von anorganisch gebundenen Gießereikernsanden. Giesserei, 96. year, No. 11, pp. 40-44. Germany.

\section{Paper number: M2014100}

Copyright (C) 2014. Published by Manufacturing Technology. All rights reserved. 